\title{
The Finite-Sample Bias of the Autoregressive Parameter Under Rank-based Estimation
}

\author{
Steven Cook \\ Department of Economics, University of Wales Swansea \\ Singleton Park, Swansea SA2 8PP, Wales
}

\begin{abstract}
Monte Carlo simulation is employed to examine the finite-sample properties of the estimated autoregressive parameter associated with the Dickey-Fuller and rank-based Dickey-Fuller unit root tests. While the downward bias associated with estimator under the Dickey-Fuller test has long been noted in the literature, the corresponding properties for the rank-based test have not been considered previously. The results presented show that in comparison to the DF test, increased bias is present for the rank-based test.
\end{abstract}

Key words: Unit Roots, Rank-Based Testing, Bias

\section{INTRODUCTION}

Following the seminal study of [1], testing of the unit root hypothesis using DF tests has become a familiar feature of applied research in econometrics and time series analysis. In its simplest form, a series $y_{t}$ is said to be a unit root process if the coefficient ? = 1 in the autoregressive representation below:

$\mathrm{y}_{\mathrm{t}}=? \mathrm{y}_{\mathrm{t}-1}+\mathrm{u}_{\mathrm{t}}$

where $u_{t}$ is an i.i.d. error term with a zero mean and constant variance. Since the initial study of DF, a range of alternative unit root tests have been proposed to examine the unit root hypothesis. Amongst these is the rank-based DF test of [2]. The use of rank-based estimation has obvious appeal as it is recognised to result in robustness to a range of problems. However, the results of GH show also show the rank-based unit root test including an intercept term to exhibit greater power than the corresponding DF test. In this study the properties of rank-based unit root test are further examined. It is known that the estimator of the autoregressive parameter ? is subject to finite-sample (downward) bias under ordinary least squares estimation (OLS) when applying DF tests [3,4]. In the present analysis, the finite-sample properties of the rank-based estimator of ? are considered.

DF and Rank-based DF Tests: Considering 'with intercept' specifications of unit root tests, the DF test examines the unit root hypothesis using the null $\mathrm{H}_{0}$ : $\beta=$ 1 in the following regression:

$\mathrm{y}_{\mathrm{t}}=\mathrm{a}+\beta \mathrm{y}_{\mathrm{t}-1}+\mathrm{e}_{\mathrm{t}}$

$\mathrm{t}=1, \ldots, \mathrm{T}$ where $T$ denotes the sample size employed. The rankbased DF test proposed by GH simply replaces $y_{t}$ with $r_{t}$, where $r_{t}$ denotes the rank of $y_{t}$ in $y_{0}, \ldots, y_{T}$. The unit hypothesis is therefore tested via the null $\mathrm{H}_{0}: \mathrm{d}=1$ in the following regression:

$\mathrm{r}_{\mathrm{t}}=?+\mathrm{d} \mathrm{r}_{\mathrm{t}-1}+\mathrm{v}_{\mathrm{t}}$

$\mathrm{t}=1, \ldots, \mathrm{T}$

However, the OLS estimator $\hat{\beta}$ is known to be biased downwards in finite-samples. In the present analysis, the nature of any bias associated with $\hat{\delta}$ is examined.

Monte Carlo Experimentation: To examine the properties of alternative estimators of the autoregressive parameter, the unit root process of (1) is numerically simulated for a range of values of ? and $T$. The specific values employed are $?=\{0.80,0.85,0.90,0.95,0.99\}$ and $T=\{50,100,250,500,1000\}$. All experiments are performed over 25,000 replications using the GAUSS programming language. The error term $u_{t}$ is generated as a standard normal variate using the RNDNS procedure. For each experimental design, the DF and rank-based DF tests of (2) and (3) are performed. In addition to noting the expected value of the estimators $B$ and $\mathrm{d}$ across replications, the expected values of the estimated standard errors, $\mathrm{SE}($.$) , of these estimators are$ also recorded. The results obtained from this analysis are presented in Table 1 . It can be seen that as expected, downward bias is associated with $\hat{\beta}$. However, while the bias increases as ? approaches 1, the bias is smaller for larger sample sizes. For example, when $?=0.95$, the bias is 0.097 when $T=50$, but is only 0.04 when $T=1000$. 
Journal of Mathematics and Statistics 1 (1): 1-2, 2005

ISSN 1549-3644

(C) Science Publications, 2005

Table 1: DF and Rank-Based DF Test Results

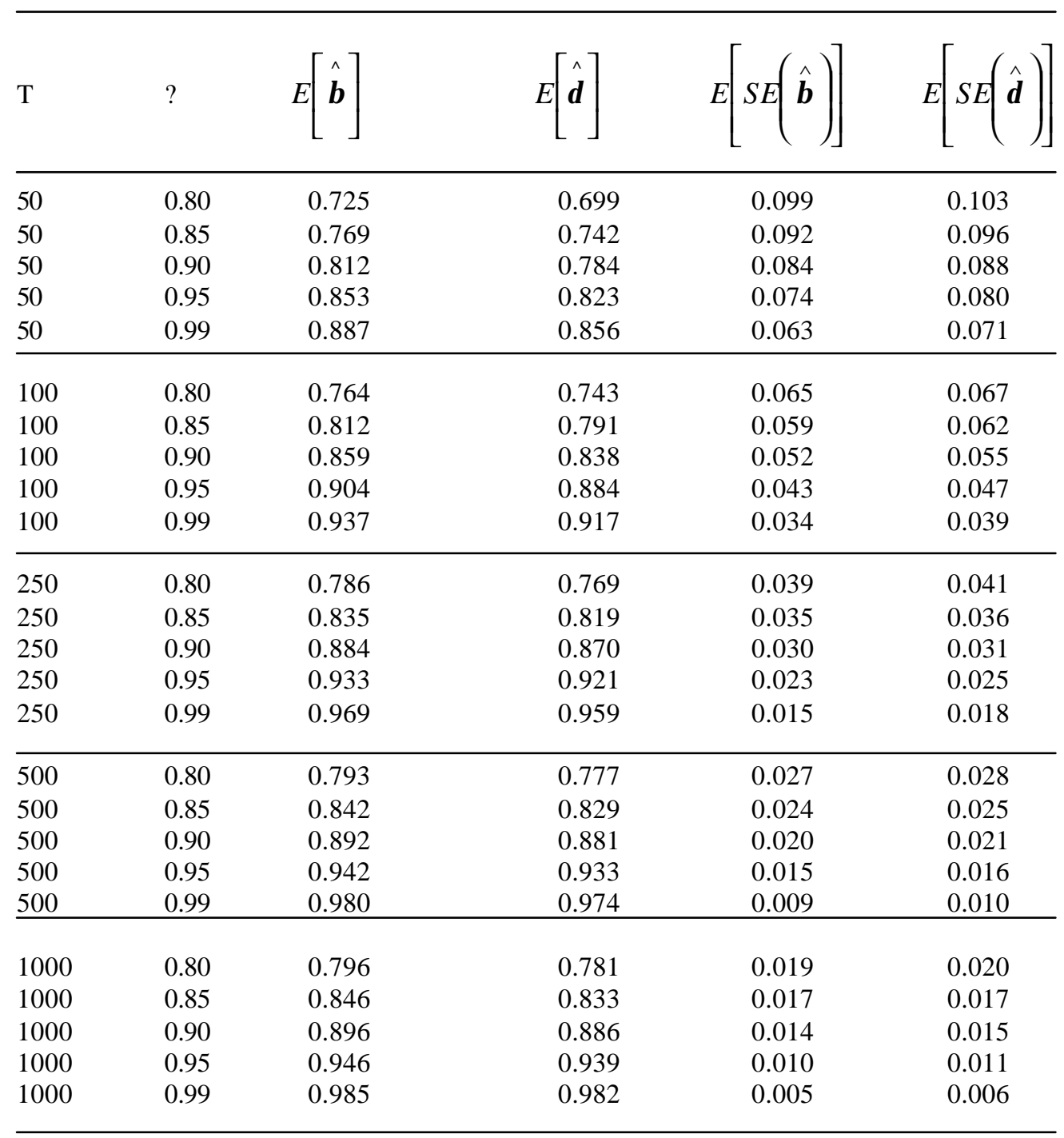

Considering the results for the rank-based test, it is clear that for all experimental designs considered, $\hat{\delta}$ exhibits greater downward bias than $\hat{\beta}$. This is perhaps surprising given the higher power of the rankbased test, but is explained by the larger standard errors associated with the estimator.

\section{CONCLUSION}

In this study the finite-sample bias of the autoregressive parameter has been examined under the rank-based DF test of [2]. The results obtained showed the downward bias of the estimator associated with this test to exceed that noted previously in the literature for the DF test. The standard errors of the estimators were also noted, and it was found that the standard errors for the rankbased test exceeded those of the DF test. However, despite underestimating the autoregressive parameter, the increased power of the rank-based test noted in the literature results can be explained by the large standard error, or variability, associated with the estimator.

\section{REFERENCES}

1. Dickey, D. and W. Fuller, 1979. Distribution of the estimators for autoregressive time series with a unit root. J. American Stat. Assoc., 74: 427-431.

2. Granger, C. and J. Hallman, 1991. Nonlinear transformations of integrated time series. J. Time Series Analysis, 12: 207-218.

3. Shaman, P. and R. Stine, 1988. The bias of autoregressive coefficient estimators. J. American Stat. Assoc., 83: 842-848.

4. Tanaka, K., 1984. An asymptotic expansion associated with maximum likelihood estimators in ARMA models. J. Royal Stat. Soc., 46: 58-67. 